\title{
Musical Descriptions based on Formal Concept Analysis and Mathematical Morphology
}

\author{
Carlos Agon ${ }^{1}$, Moreno Andreatta ${ }^{1,2}$, Jamal Atif $^{3}$, Isabelle Bloch ${ }^{4}$, and Pierre \\ Mascarade $^{3}$ \\ 1 CNRS-IRCAM-UPMC, Paris, France carlos.agon@ircam.fr, \\ moreno.andreatta@ircam.fr \\ 2 IRMA/GREAM/USIAS, Université de Strasbourg, Paris, France \\ 3 Université Paris-Dauphine, PSL Research University, CNRS, UMR 7243, \\ LAMSADE, 75016 Paris, France, \\ jamal.atif@dauphine.fr,pierre.m@protonmail.com \\ 4 LTCI, Téécom ParisTech, Université Paris-Saclay, Paris, France, \\ isabelle.bloch@telecom-paristech.fr
}

\begin{abstract}
In the context of mathematical and computational representations of musical structures, we propose algebraic models for formalizing and understanding the harmonic forms underlying musical compositions. These models make use of ideas and notions belonging to two algebraic approaches: Formal Concept Analysis (FCA) and Mathematical Morphology (MM). Concept lattices are built from interval structures whereas mathematical morphology operators are subsequently defined upon them. Special equivalence relations preserving the ordering structure of the lattice are introduced in order to define musically relevant quotient lattices modulo congruences. We show that the derived descriptors are well adapted for music analysis by taking as a case study Ligeti's String Quartet No. 2.
\end{abstract}

Keywords: Computational music analysis, Formal concept analysis, Mathematical morphology, Congruences, Quotient lattices, Harmonico-morphological descriptors, Musical Information Research

\section{Introduction}

Despite a long historical relationship between mathematics and music, computational music analysis is a relatively recent research field. In contrast to statistical methods and signal-based approaches currently employed in Music Information Research (or MIR ${ }^{5}$ ), the paper at hand stresses the necessity of introducing a structural multidisciplinary approach into computational musicology making use of advanced mathematics. It is based on the interplay between algebra and topology and opens promising perspectives on important prevailing challenges,

\footnotetext{
${ }^{5}$ Following the Roadmap described in [18], we prefer to consider MIR as the field of Music Information Research instead of limiting the scope to purely Music Information Retrieval.
} 
such as the formalization and representation of musical structures and processes, or the automatic classification of musical styles. It also differs from traditional applications of mathematics to music in aiming to build bridges between different musical genres, ranging from contemporary art music to popular music, and therefore showing the generality of the conceptual tools introduced into computational musicology. Most of these tools belong to the domain of Formal Concept Analysis (FCA), a research field that has been introduced in the beginning of the 1980s by Rudolf Wille and, independently, by Marc Barbut and Louis Frey [3], in both cases as an attempt at reconstructing Lattice Theory $[20,23] .{ }^{6}$ Interestingly, music was a major inspirational field for applying formal concept analysis, within the Darmstadt tradition. In his introductory essay on the link between mathematics and music [22], R. Wille proposed to represent the chords of the diatonic scale as equivalence classes, leading to a chord concept lattice. Following this seminal work, a morphology of chords has been proposed by T. Noll [13], showing the interest of this approach for computational music analysis. Some recent work renewed the interest of this approach as an original way to represent musical structures within the field of Music Information Research, by stressing the underlying algebraic aspects, with application of ordered structures and concept lattices to the algebraic enumeration and classification of musical structures for computational music analysis $[17,16] .^{7}$

In this paper, we propose a way to combine algebraic formalizations and lattice-based representations of harmonic structures within existing musical compositions. Instead of analyzing the musical pitch content, ${ }^{8}$ formal concept lattices are built from intervallic structures. The objective is to provide a way of summarizing the harmonic musical content by studying the properties of the underlying lattice organization. We make use of operators belonging to Mathematical Morphology (MM) which are defined on such lattices. This enables to define congruences between elements of the lattice and associated quotient lattices. These quotient lattices are in fact the symbolic and structural descriptors of the musical pieces that we propose to use as a generic tool for computational music analysis. As a case study, we show how the first movement of Ligeti's String Quartet No. 2 can be compactly described with three of such quotient lattices.

This paper is organized as follows. In Section 2 we summarize previous work on the definition of lattices of harmonic structures by means of their interval content. In Section 3 we recall some definitions of mathematical morphology on complete lattices and propose specific operators (dilation and erosion) on musical concept lattices. The main original contribution of this paper is contained in

\footnotetext{
${ }^{6}$ See [21] for an interesting discussion on the mutual influences between the Darmstadt school on Formal Concept Analysis and the French tradition on Treillis de Galois.

7 See the Mutabor language (http://www.math.tu-dresden.de/ mutabor/) for a music programming language making use of the FCA-based Standard Language for Music Theory [12] originally conceived by Rudolf Wille and currently developed at the University of Dresden.

${ }^{8}$ Note that, at this stage, the time information is not taken into account, and a musical excerpt is considers as an unordered set of chords.
} 
Section 4 where we define a way to reduce a given concept lattice to its "core" structure via congruence relations. The resulting quotient lattices are precisely the structural descriptors used in the representation of a given musical piece. This opens new challenging perspectives for automatic music analysis and structural comparison between musical pieces of different styles.

\section{Lattice of interval structures}

In this section we recall how a concept lattice can be built from harmonic forms (as objects) and intervals (as attributes) $[16,17]$.

Definition 1 (Harmonic system) Let $T$ be a set, $\mathrm{I}=(I,+,-, 0)$ an Abelian group, and $\Delta: T \times T \rightarrow I$ a mapping such that $\forall t_{1}, t_{2}, t_{3} \in T$ :

$$
\Delta\left(t_{1}, t_{2}\right)+\Delta\left(t_{2}, t_{3}\right)=\Delta\left(t_{1}, t_{3}\right) \quad \text { and } \quad \Delta\left(t_{1}, t_{2}\right)=0 \text { iff } t_{1}=t_{2} .
$$

Then the triplet $\mathbb{T}=(T, \Delta, \mathrm{I})$ is called algebraic harmonic system. Elements of $T$ are tones and any subset of $T$ is a chord. Elements of I are musical intervals.

Here we consider $\mathbb{T}_{n}=\left(\mathbb{Z}_{n}, \Delta_{n}, \mathbb{Z}_{n}\right)$, where $n \in \mathbb{Z}_{+}$represents an octave, $\mathbb{Z}_{n}=$ $\mathbb{Z} / n \mathbb{Z}$, and $\Delta_{n}$ is the difference modulo $n$. All chords are then projected in $\mathbb{T}_{n}$ using a canonical homomorphism. Moreover, two chords having the same number of notes (or chromas) and the same intervals between notes (i.e. defined up to a transposition) are considered equivalent, thus defining harmonic forms.

Definition 2 (Harmonic forms) The set $\mathcal{H}\left(\mathbb{T}_{n}\right)$ of the harmonic forms of $\mathbb{T}_{n}$ is composed by the equivalence classes of the following equivalence relation $\Psi$ :

$$
\forall H_{1} \subseteq \mathbb{Z}_{n}, \forall H_{2} \subseteq \mathbb{Z}_{n}, H_{1} \Psi H_{2} \text { iff } \exists i \mid H_{1}=H_{2}+i
$$

where $H+i=\{t+i \mid t \in H\}$ if $t+i$ exists for all $t \in H$.

In the sequel, we will use the following notation: $I_{H, t}=\left\{\Delta\left(t, t^{\prime}\right) \mid t^{\prime} \in H\right\}$.

Definition 3 (Musical formal context) A musical formal context, denoted by $\mathbb{K}=\left(\mathcal{H}\left(\mathbb{T}_{n}\right), \mathbb{Z}_{n}, R\right)$ is defined by considering harmonic forms, in $G=\mathcal{H}\left(\mathbb{T}_{n}\right)$, as objects and intervals, in $M=\mathbb{Z}_{n}$, as attributes. The relation $R$ is defined from the occurrence of an interval in an harmonic form. A formal concept is a pair $(X, Y), X \subseteq G, Y \subseteq M$ such that $X \times Y \subseteq R$ and that is maximal for this property. The concept lattice $(\mathbb{C}(\mathbb{K}), \preceq)$ is then defined from the formal context and the partial ordering $\preceq$ defined as:

$$
\left(X_{1}, Y_{1}\right) \preceq\left(X_{2}, Y_{2}\right) \Leftrightarrow X_{1} \subseteq X_{2}\left(\Leftrightarrow Y_{2} \subseteq Y_{1}\right) .
$$

For $X \subseteq G$ and $Y \subseteq M$, the derivation operators $\alpha$ and $\beta$ are defined as $\alpha(X)=$ $\{m \in M \mid \forall g \in X,(g, m) \in R\}$, and $\beta(Y)=\{g \in G \mid \forall m \in Y,(g, m) \in R\}$. The pair $(\alpha, \beta)$ induces a Galois connection between the partially ordered power sets 
$(\mathcal{P}(G), \subseteq)$ and $(\mathcal{P}(M), \subseteq)$, i.e. $X \subseteq \alpha(Y)$ iff $Y \subseteq \beta(X)$. Then the pair $(X, Y)$ (with $X \subseteq G$ and $Y \subseteq M$ ) is a formal concept if and only if $\alpha(X)=Y$ and $\beta(Y)=X$ ( $X$ is then called extend and $Y$ intend of the formal concepts).

As in any concept lattice, the supremum and infimum of a family of concepts $\left(X_{t}, Y_{t}\right)_{t \in T}$ are:

$$
\begin{aligned}
& \wedge_{t \in T}\left(X_{t}, Y_{t}\right)=\left(\cap_{t \in T} X_{t}, \alpha\left(\beta\left(\cup_{t \in T} Y_{t}\right)\right)\right) \\
& \vee_{t \in T}\left(X_{t}, Y_{t}\right)=\left(\beta\left(\alpha\left(\cup_{t \in T} X_{t}\right)\right), \cap_{t \in T} Y_{t}\right),
\end{aligned}
$$

Example 1. As a running example in this paper, we consider 7-tet $\mathbb{T}_{7}$ (e.g. the diatonic scale $\mathrm{C}, \mathrm{D}, \mathrm{E}, \mathrm{F}, \mathrm{G}, \mathrm{A}, \mathrm{B})$. Let us define the formal context $\mathbb{K}=$ $\left(\mathcal{H}\left(\mathbb{T}_{7}\right), \mathbb{Z}_{7}, R\right)$, where $R$ is a binary relation such that for any harmonic form $F \in \mathcal{H}\left(\mathbb{T}_{7}\right)$ and any interval $i \in \mathbb{Z}_{7}$, we have $(F, i) \in R$ iff there exists $t \in F$ such that $i \in I_{F, t}$. Intervals are denoted by the index of the last note from the starting one, hence for the 7-tet intervals are unison (0), second (1), third (2), fourth (3). Note that other intervals $(4,5$, and 6$)$ are derived from these basic ones by group operations (inversion modulo octave).

Figure 1 illustrates the formal context $\mathbb{K}=\left(\mathcal{H}\left(\mathbb{T}_{7}\right), \mathbb{Z}_{7}, R\right)$ and the concept

\begin{tabular}{|c|c|c|c|c|}
\hline & 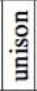 & 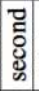 & . 글 & 馬 \\
\hline \multicolumn{5}{|l|}{ rest } \\
\hline 0 & $x$ & & & \\
\hline 0,1 & $x$ & $x$ & & \\
\hline 0,2 & $x$ & & $x$ & \\
\hline $0,1,2$ & $x$ & $x$ & $x$ & \\
\hline 0,3 & $x$ & & & $x$ \\
\hline $0,1,3$ & $x$ & $x$ & $x$ & $x$ \\
\hline $0,2,3$ & $x$ & $x$ & $x$ & $x$ \\
\hline $0,1,2,3$ & $x$ & $x$ & $x$ & $x$ \\
\hline $0,1,4$ & $x$ & $x$ & & $x$ \\
\hline $0,2,4$ & $x$ & & $x$ & $x$ \\
\hline $0,1,2,4$ & $x$ & $x$ & $x$ & $x$ \\
\hline $0,1,3,4$ & $x$ & $\times$ & $\times$ & $x$ \\
\hline $0,2,3,4$ & $x$ & $x$ & $x$ & $x$ \\
\hline $0,1,2,3,4$ & $x$ & $\times$ & $\times$ & $x$ \\
\hline $0,1,3,5$ & $x$ & $\times$ & $x$ & $x$ \\
\hline $0,1,2,3,5$ & $x$ & $x$ & $\times$ & $x$ \\
\hline $0,1,2,4,5$ & $x$ & $\times$ & $\times$ & $x$ \\
\hline $0,1,2,3,4,5$ & $x$ & $\times$ & $x$ & $x$ \\
\hline $0,1,2,3,4,5,6$ & $x$ & $\times$ & $x$ & $x$ \\
\hline
\end{tabular}
lattice $(\mathbb{C}(\mathbb{K}), \preceq)$ as defined in Definition 3 .

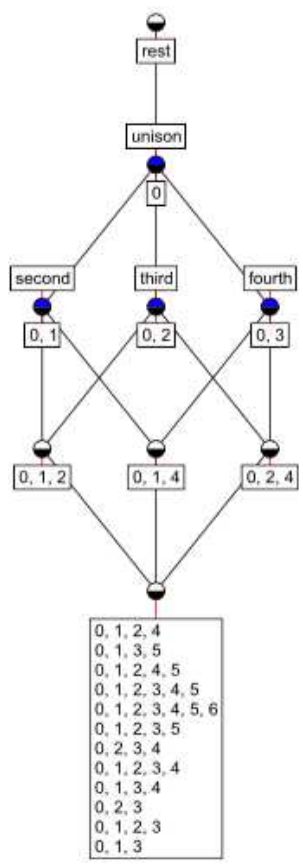

Fig. 1. Formal context $\mathbb{K}=\left(\mathcal{H}\left(\mathbb{T}_{7}\right), \mathbb{Z}_{7}, R\right)$ and concept lattice $(\mathbb{C}(\mathbb{K}), \preceq)$ (reproduced from $[17])$. 


\section{Mathematical morphology operations on musical concept lattices}

\subsection{Preliminaries}

Let us recall the algebraic framework of mathematical morphology. Let $(\mathcal{L}, \preceq)$ and $\left(\mathcal{L}^{\prime}, \preceq^{\prime}\right)$ be two complete lattices (which do not need to be equal). All the following definitions and results are common to the general algebraic framework of mathematical morphology in complete lattices $[4,5,7,8,11,15,19]$. Note that different terminologies can be found in different lattice theory related contexts (refer to [14] for equivalence tables).

Definition 4 An operator $\delta: \mathcal{L} \rightarrow \mathcal{L}^{\prime}$ is an algebraic dilation if it commutes with the supremum (sup-preserving mapping):

$$
\forall\left(x_{i}\right) \in \mathcal{L}, \delta\left(\vee_{i} x_{i}\right)=\vee_{i}^{\prime} \delta\left(x_{i}\right),
$$

where $\vee\left(\right.$ respectively $\left.\vee^{\prime}\right)$ denotes the supremum associated with $\preceq$ (respectively $\left.\preceq^{\prime}\right)$.

An operator $\varepsilon: \mathcal{L}^{\prime} \rightarrow \mathcal{L}$ is an algebraic erosion if it commutes with the infimum (inf-preserving mapping):

$$
\forall\left(x_{i}\right) \in \mathcal{L}^{\prime}, \varepsilon\left(\wedge_{i}^{\prime} x_{i}\right)=\wedge_{i} \varepsilon\left(x_{i}\right),
$$

where $\wedge$ and $\wedge^{\prime}$ denote the infimum associated with $\preceq$ and $\preceq^{\prime}$, respectively.

This general definition allows defining mathematical morphology operators such as dilations and erosions in many types of settings, such as sets, functions, fuzzy sets, rough sets, graphs, hypergraphs, various logics, etc., based on their corresponding lattices.

Algebraic dilations $\delta$ and erosions $\varepsilon$ are increasing operators; moreover $\delta$ preserves the smallest element and $\varepsilon$ preserves the largest element.

A fundamental notion in this algebraic framework is the one of adjunction.

Definition 5 A pair of operators $(\varepsilon, \delta), \delta: \mathcal{L} \rightarrow \mathcal{L}^{\prime}, \varepsilon: \mathcal{L}^{\prime} \rightarrow \mathcal{L}$, defines an adjunction if

$$
\forall x \in \mathcal{L}, \forall y \in \mathcal{L}^{\prime}, \delta(x) \preceq^{\prime} y \Longleftrightarrow x \preceq \varepsilon(y) .
$$

Note that the notion of adjunction corresponds to the Galois connection by reversing the order of either $\mathcal{L}$ or $\mathcal{L}^{\prime}$. This induces a first direct link between derivation operators $\alpha, \beta$ on the one hand, and $\delta, \varepsilon$ on the other hand. Further links between FCA and MM have been investigated in $[1,2]$.

Some important properties, that will be used in the following, are summarized as follows.

Proposition 1 [e.g. $[8,15]]$ If a pair of operators $(\varepsilon, \delta)$ defines an adjunction, then the following results hold:

$-\delta$ preserves the smallest element and $\varepsilon$ preserves the largest element; 
- $\delta$ is a dilation and $\varepsilon$ is an erosion (in the sense of Definition 4).

Let $\delta$ and $\varepsilon$ be two increasing operators such that $\delta \varepsilon$ is anti-extensive and $\varepsilon \delta$ is extensive. Then $(\varepsilon, \delta)$ is an adjunction.

The following representation result also holds. If $\varepsilon$ is an increasing operator, it is an algebraic erosion if and only if there exists $\delta$ such that $(\varepsilon, \delta)$ is an adjunction. The operator $\delta$ is then an algebraic dilation and can be expressed as $\delta(x)=\wedge^{\prime}\left\{y \in \mathcal{L}^{\prime} \mid x \preceq \varepsilon(y)\right\}$. A similar representation result holds for erosion.

All these results hold in the particular case of a concept lattice.

Particular forms of dilations and erosions can be defined based on the notion of structuring element, which can be a neighborhood relation or any binary relation $[5,19]$. In particular, such structuring elements can be defined as the balls of a given distance. This has been investigated in concept lattices, using several distances, in $[1,2]$.

In the next sections we describe two examples of dilations and erosions, defined on the lattice $\mathbb{C}$, used to handle musical format contexts. They have been implemented in SageMath. ${ }^{9}$ The other definitions proposed in $[1,2]$ could be exploited as well for musical concept lattices.

\subsection{Dilations and erosions from the decomposition into join or meet irreducible elements}

The first example relies on the decomposition of a concept in a join-irreducible form (hence suitable for defining dilations), respectively meet-irreducible for defining erosions.

Definition 6 (Join and meet irreducible element) An element a of a lattice $\mathbb{C}$ is join (respectively meet) irreducible if it is not equal to the least element of the lattice (respectively the largest element) and $\forall(a, b) \in \mathbb{C}^{2}, a=b \vee c \Rightarrow a=$ $b$ or $a=c$ (respectively $a=b \wedge c \Rightarrow a=b$ or $a=c)$.

Any element of the lattice can be written (usually not uniquely) as the join (respectively meet) of some irreducible elements.

Since a dilation (respectively erosion) is defined as an operator that commutes with the supremum (respectively infimum), it is sufficient to define these operators on join (respectively meet) irreducible elements to extend them to any element of the lattice. This will be exploited next, in the proposed algorithm in Section 4.

\subsection{Dilations and erosions based on structuring elements derived from a valuation}

In the second example, we define dilations and erosions, based on structuring elements that are balls of a distance derived from a valuation on the lattice. In the following we propose to use valuations defined as the cardinality of filters or ideals.

\footnotetext{
${ }^{9}$ http://www.sagemath.org/
} 
Definition 7 (Filter and ideal) Let a be an element of a lattice $\mathbb{C}$. The filter and ideal associated with a are the subsets of $\mathbb{C}$ defined as:

$$
\begin{aligned}
& F(a)=\{b \in \mathbb{C} \mid a \preceq b\} \\
& I(a)=\{b \in \mathbb{C} \mid b \preceq a\}
\end{aligned}
$$

Definition 8 Let $(\mathbb{C}, \preceq)$ be a concept lattice. A real-valued function w on $(\mathbb{C}, \preceq)$ is a lower valuation if it satisfies the following (supermodular) property:

$$
\forall\left(a_{1}, a_{2}\right) \in \mathbb{C}^{2}, w\left(a_{1}\right)+w\left(a_{2}\right) \leq w\left(a_{1} \wedge a_{2}\right)+w\left(a_{1} \vee a_{2}\right),
$$

and is an upper valuation if it satisfies the following (submodular) property:

$$
\forall\left(a_{1}, a_{2}\right) \in \mathbb{C}^{2}, w\left(a_{1}\right)+w\left(a_{2}\right) \geq w\left(a_{1} \wedge a_{2}\right)+w\left(a_{1} \vee a_{2}\right)
$$

A real-valued function is increasing (isotone) if $a_{1} \preceq a_{2}$ implies $w\left(a_{1}\right) \leq w\left(a_{2}\right)$ and decreasing (antitone) if $a_{1} \preceq a_{2}$ implies $w\left(a_{1}\right) \geq w\left(a_{2}\right)$.

Proposition $2([\mathbf{9}, \mathbf{1 0}])$ Let $w$ be a real-valued function on a concept lattice $(\mathbb{C}, \preceq)$. Then the function defined as:

$$
\forall\left(a_{1}, a_{2}\right) \in \mathbb{C}^{2}, d_{w}\left(a_{1}, a_{2}\right)=2 w\left(a_{1} \wedge a_{2}\right)-w\left(a_{1}\right)-w\left(a_{2}\right)
$$

is a pseudo-metric if and only if $w$ is a decreasing upper valuation.

The function defined as:

$$
\forall\left(a_{1}, a_{2}\right) \in \mathbb{C}^{2}, d_{w}\left(a_{1}, a_{2}\right)=w\left(a_{1}\right)+w\left(a_{2}\right)-2 w\left(a_{1} \vee a_{2}\right)
$$

is a pseudo-metric if and only if $w$ is a decreasing lower valuation.

Proposition 3 (Valuation from a filter or ideal) Let $w_{F}$ be the mapping defined on a concept lattice $\mathbb{C}$ as $\forall a \in \mathbb{C}, w_{F}(a)=|F(a)|$ where $F$ is the filter associated with $a$. Then $w_{F}$ is a decreasing lower valuation, i.e.

$$
\forall\left(a_{1}, a_{2}\right) \in \mathbb{C}^{2}, w_{F}\left(a_{1}\right)+w_{F}\left(a_{2}\right) \leq w_{F}\left(a_{1} \wedge a_{2}\right)+w_{F}\left(a_{1} \vee a_{2}\right)
$$

The mapping $d$ from $\mathbb{C} \times \mathbb{C}$ into $\mathbb{R}+$ defined as $\forall\left(a_{1}, a_{2}\right) \in \mathbb{C}^{2}, d\left(a_{1}, a_{2}\right)=$ $w_{F}\left(a_{1}\right)+w_{F}\left(a_{2}\right)-2 w_{F}\left(a_{1} \vee a_{2}\right)$ is therefore a pseudo-distance.

Similarly, a pseudo-distance can be defined from the cardinality of the ideals.

Once the distance is defined, a structuring element is defined as a ball of this distance, for a given radius $n$. Dilations and erosions then write:

$$
\begin{gathered}
\forall A \subseteq \mathbb{C}, \delta(A)=\{b \in \mathbb{C} \mid d(b, A) \leq n\} \\
\forall A \subseteq \mathbb{C}, \varepsilon(A)=\{b \in \mathbb{C} \mid d(b, \mathbb{C} \backslash A)>n\}=\mathbb{C} \backslash \delta(\mathbb{C} \backslash A)
\end{gathered}
$$

Note that in the first example, such dilations and erosions can also be applied to irreducible elements, in order to derive dilations and erosions using the commutativity with the supremum or infimum, as will be used in Section 4. 


\section{Harmonico-morphological descriptors based on congruence relations}

By using the concepts we have previously introduced, we define a way of reducing a concept lattice via some equivalence relations, namely congruences. This ideas goes back to Birkhoff [4], and was used in several works such as [6] with extension to non transitive relations (tolerance relations). Here propose new congruences based on mathematical morphology.

\subsection{Definitions}

Definition 9 (Congruences and quotient lattices) An equivalence relation $\theta$ on a lattice $\mathcal{L}$ is a congruence if it is compatible with join and meet, i.e. $(\theta(a, b)$ and $\theta(c, d)) \Rightarrow(\theta(a \vee c, b \vee d)$ and $\theta(a \wedge c, b \wedge d))$, for all $a, b, c, d \in \mathcal{L}$. This equivalence relation allows defining a quotient lattice which will be denoted as $\mathcal{L} / \theta$.

Hence $\vee$ and $\wedge$ induce joint and meet operators on the quotient lattice $\mathcal{L} / \theta$. By denoting $[a]_{\theta}$ the equivalence class of $a \in \mathcal{L}$ for $\theta$, we have $[a]_{\theta} \vee[b]_{\theta}=[a \vee b]_{\theta}$, and a similar relation for $\wedge$ (note that the same notations are used on $\mathcal{L}$ and on $\mathcal{L} / \theta$ for meet and join, when no ambiguity occurs). This way of defining quotient lattices enables to transfer the structure from the original concept lattice to the reduced one, therefore preserving the order relations between the elements.

Example 2. Let us apply these notions to the concept lattice $(\mathbb{C}(\mathbb{K}), \preceq)$ associated with the musical formal concept defined from the 7-tet, as before (see Example 1). Ajouter l'explication sur la multiplicit des intervalles... In this diatonic space, one may define a formal equivalence relation between major/minor chords and major/minor seventh chords allowing us to reduce the initial lattice to the corresponding quotient lattice. More precisely, the congruence relation is defined in order to group $\{0,2,4\}$ and $\{0,1,3,5\}$ into the same class. The other classes are derived so as to preserve the ordering relations between concepts. This reduction process is represented in Figure 2. For instance, let us consider $\{0,1\}$ (we only mention the extend of the concepts here). We have $\{0,2,4\} \wedge\{0,1\}=\{0\}$ and $\{0,1,3,5\} \wedge\{0,1\}=\{0,1\}$, which is consistent with the fact that $\{0\}$ and $\{0,1\}$ are in the same congruence class.

Expliquer les noeuds intermediaires (deja chez Wille etc.)

The quotient lattice $\mathbb{C} / \theta$ formalizes the partition of the harmonic system, preserving its structure. A sub-lattice of $\mathbb{C}(\mathbb{K})$ isomorphic to $\mathbb{C} / \theta$ is interpreted as a harmonic sub-system compatible with the harmonic structure generated by the partition defined from a set of harmonic forms.

Example 3. An interesting congruence, from a musical point of view, can be defined by gathering the most common harmonic tonal forms in the same equivalence class (perfect chords, seventh chords and ninth chords). The generating 

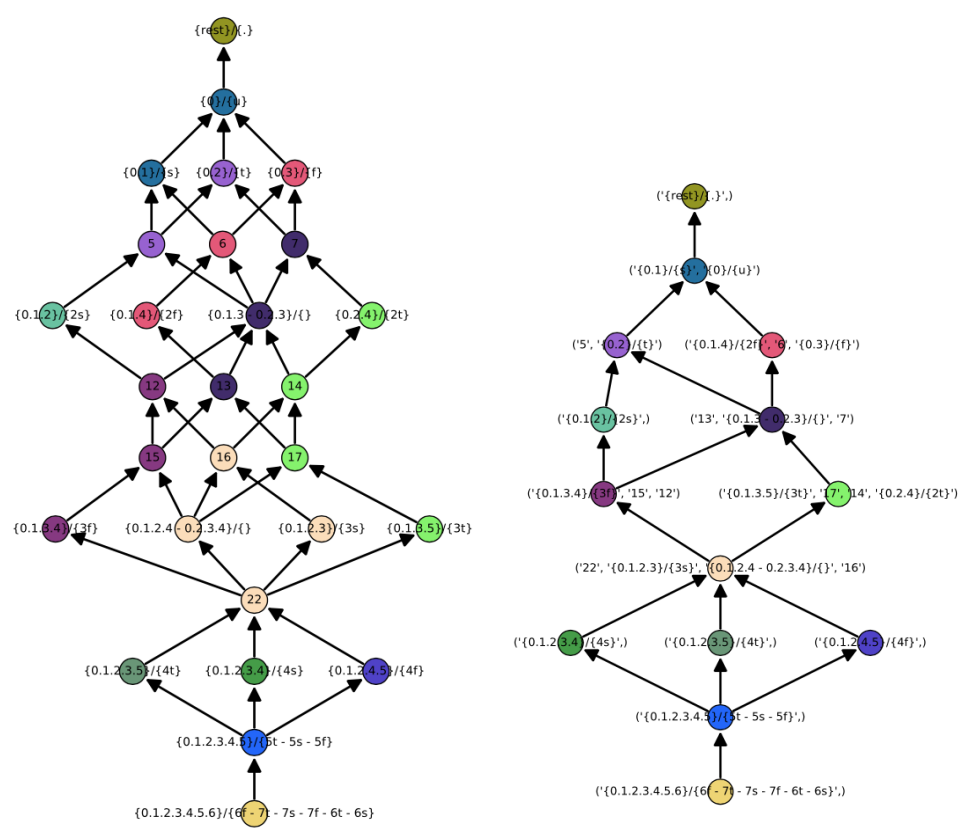

Fig. 2. Left: concept lattice, where all concepts of a same color belong to the same equivalence class according the to chosen congruence. Right: quotient lattice.

elements for this class are $\{0,2,4\},\{0,1,3,5\}$, and $\{0,1,2,3,5\}$ (again only the extend is mentioned here). Another class is generated from $\{0,3\}$ (i.e. fourths, which are also interesting from a musical point of view). The other classes are derived to preserve the ordering relations. This congruence $\theta^{*}$ is illustrated in Figure 3, still for the 7-tet, along with the corresponding quotient lattice. The first generated class in displayed in pink and the second one in green. In this case, the quotient lattice, is simply a chain, representing a linear complete ordering among equivalence classes. ${ }^{10}$ The remaining concepts (excluding the top and the bottom of the lattice) form a third equivalence class. Let us consider the following example, to illustrate the consistency of the generated classes: $\{0,3\}$ and $(\{0,1,3\},\{0,2,3\})$ are congruent (both are in the green class); similarly $\{0,1,3,5\}$ and $\{0,1,2,3\}$ are congruent (both in the pink class). The conjunctions $\{0,3\} \wedge\{0,1,3,5\}=\{0,3\}$ and $(\{0,1,3\},\{0,2,3\}) \wedge\{0,1,2,3\}=$ $(\{0,1,3\},\{0,2,3\})$ are congruent, and the disjunctions $\{0,3\} \vee\{0,1,3,5\}=$ $\{0,1,3,5\}$ and $(\{0,1,3\},\{0,2,3\}) \vee\{0,1,2,3\}=\{0,1,2,3\}$ are also congruent.

We now propose to exploit the two notions of congruence and of morphological operators to define musical descriptors.

\footnotetext{
${ }^{10}$ An interesting question, which still remains open, concerns the possible ways of generating chains which are musically relevant by carefully selecting the underlying equivalence classes.
} 

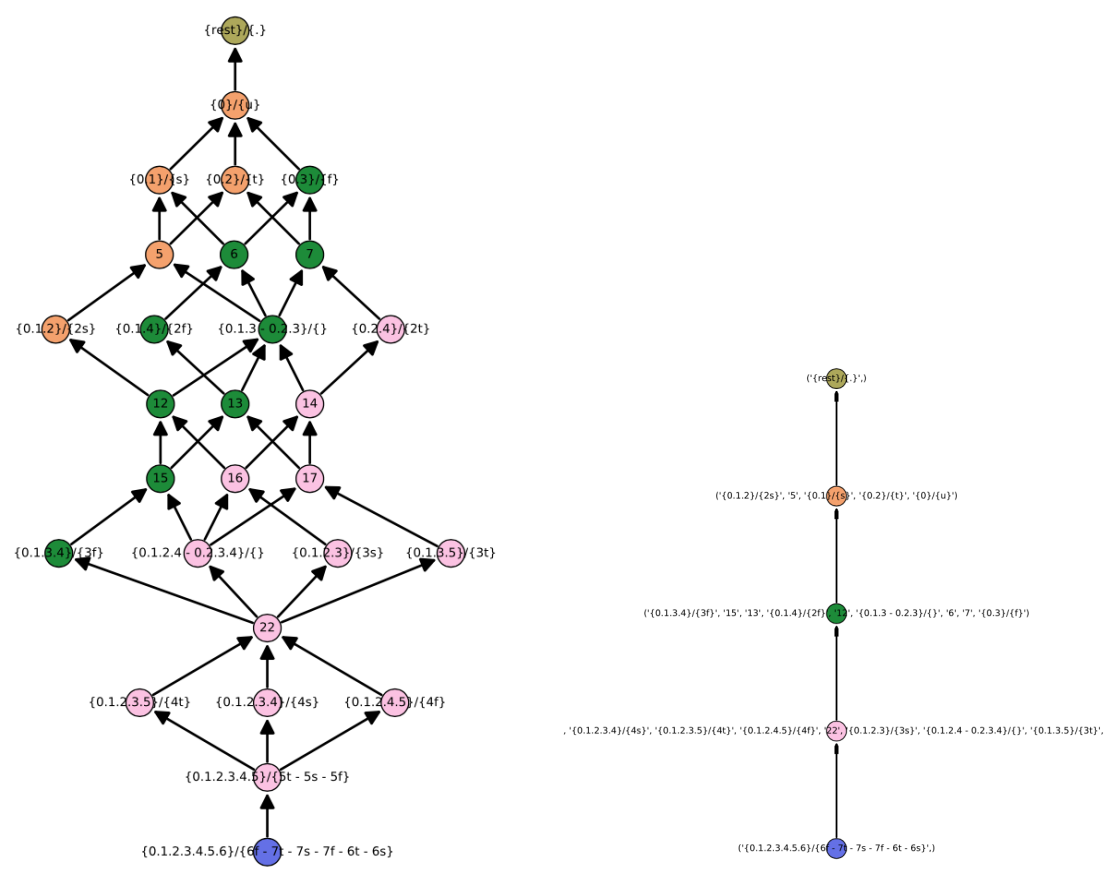

Fig. 3. Left: congruence $\theta^{*}$ on the 7 -tet concept lattice. Right: quotient lattice $\mathbb{C} / \theta^{*}$.

Definition 10 (Harmonico-morphological descriptors) Let $\mathcal{M}$ be a musical piece, $\mathbb{T}_{\mathcal{M}}$ the harmonic system associated with it, and $\mathbb{C}(\mathcal{M})$ the corresponding concept lattice. The core idea of the proposed descriptors is to use dilations and erosions of the set of formal concepts to provide upper and lower bounds of the description on the one hand, and congruences to provide a structural summary of the harmonic forms on the other hand. The set of formal concepts corresponding to the harmonic forms in $\mathcal{M}$ is denoted by $H_{\mathbb{C}}^{\mathcal{M}}$. The dilations $\delta$ and erosions $\varepsilon$ can typically be defined from a metric associated with a valuation on $\mathbb{C}(\mathcal{M})$. Three congruences are then defined:

- $\theta$ grouping all formal concepts in $H_{\mathbb{C}}^{\mathcal{M}}$ into one same class;

- $\theta_{\delta}$ grouping all formal concepts in $\delta\left(H_{\mathbb{C}}^{\mathcal{M}}\right)$ into one same class;

- $\theta_{\varepsilon}$ grouping all formal concepts in $\varepsilon\left(H_{\mathbb{C}}^{\mathcal{M}}\right)$ into one same class.

The proposed harmonic descriptors are the quotient lattices $\mathbb{C}(\mathcal{M}) / \theta, \mathbb{C}(\mathcal{M}) / \theta_{\delta}$, and $\mathbb{C}(\mathcal{M}) / \theta_{\varepsilon}$.

We argue that these descriptors are good representative of $\mathcal{M}$, since they preserve the intervallic structures, and provide compact summaries, which would allow for comparison between musical pieces. 


\subsection{Algorithm}

The procedure developed to generate the proposed descriptors is given in Algorithm 1 (implementation was done in SageMath).

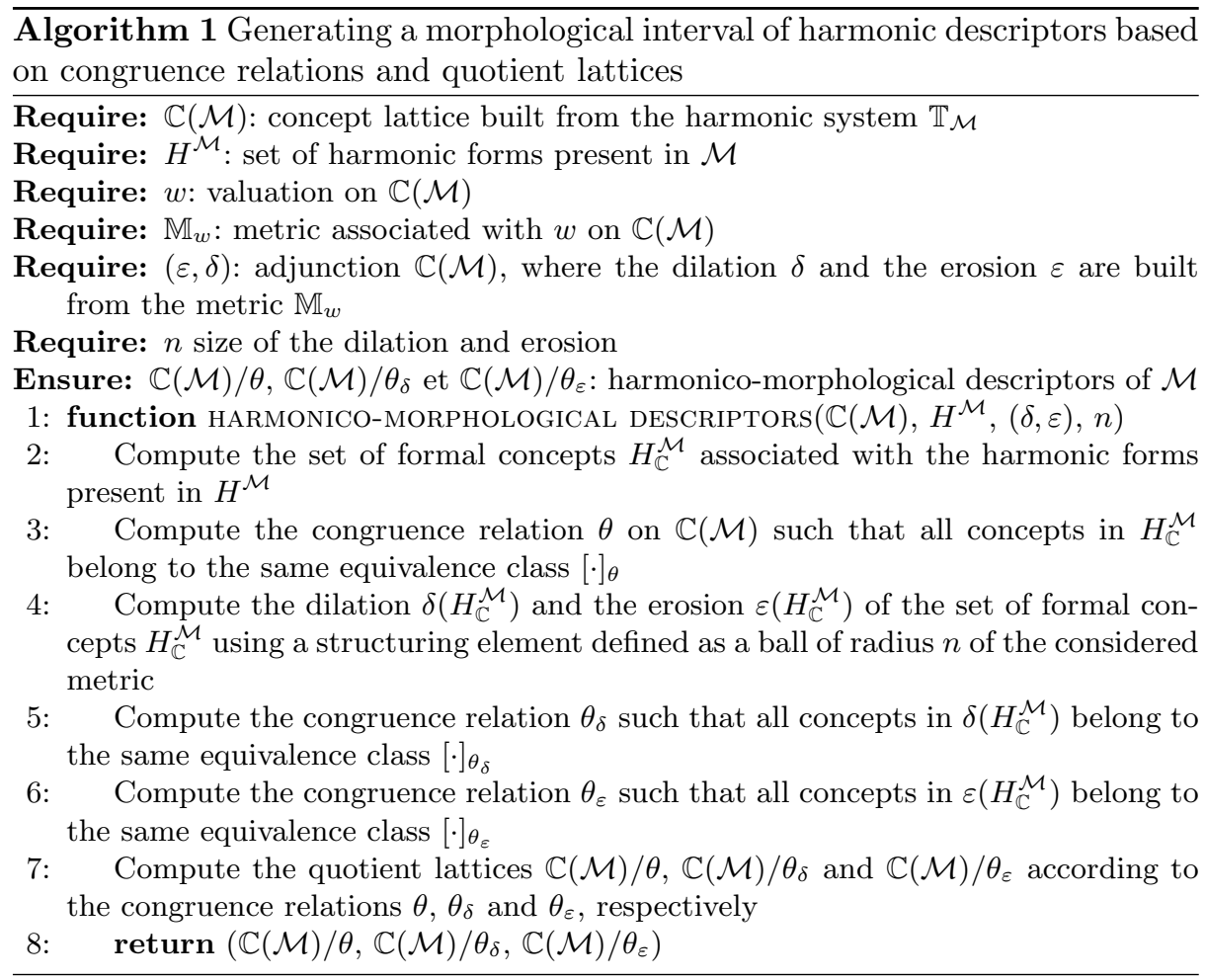

\subsection{Example on Ligeti's String Quartet No. 2}

As an illustrative example, we apply the proposed method for computing the musical descriptors on $\mathcal{M}$ corresponding to the first movement of Ligeti's String Quartet No. 2. For example, the first set, $\{0,1\}$, corresponds to the two notes chord $\{C, D\}$, whereas the last one, $\{0,1,2,3\}$, corresponds to the tetrachord $\{C, D, E, F\}$.

We use the previous 7-tet lattice for the analysis by selecting a limited number of harmonic forms which are used by the composer. These forms are given in Figure 4 by means of a circular representation corresponding to the underlying cyclic group of order 7 .

The chosen valuation is the cardinality of the filter $w_{F}$ (see Section 3 ). Note that other valuations could be used as well. The associated distance was used to defined elementary dilations and erosions (with $n=2$ ) on join-irreducible 

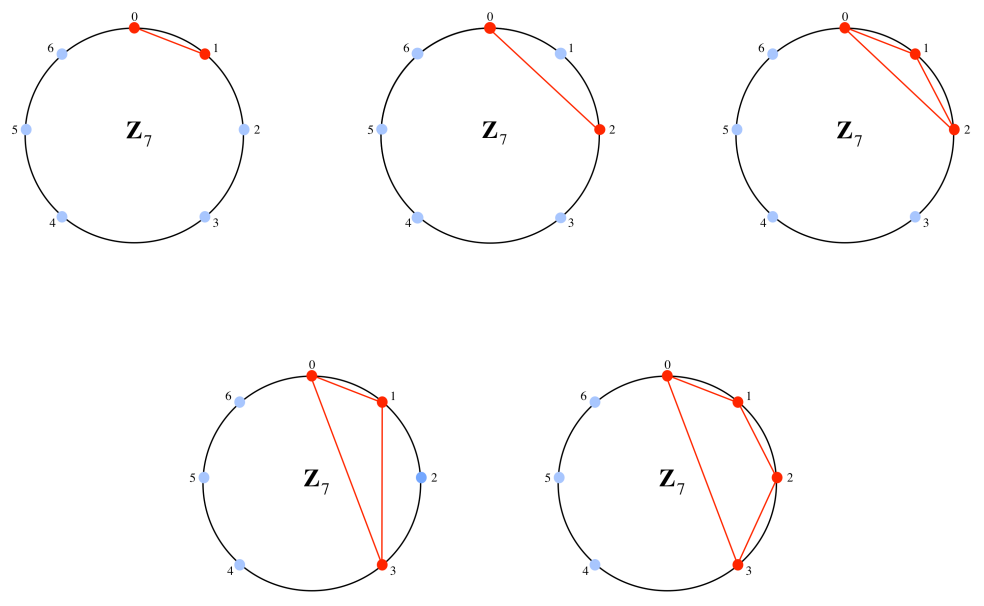

Fig. 4. Some harmonic forms in Ligeti's quartet fragment.

(respectively meet-irreducible) elements. The dilation or erosion of any concept is then derived from its decomposition into irreducible elements and using the commutativity with the supremum, respectively the infimum.

The steps of Algorithm 1 for this fragment are illustrated in Figures 5-7. As we could guess from the congruence relations, the final quotient lattices show isomorphic relations between $\mathbb{C}(\mathcal{M}) / \theta$ and $\mathbb{C}(\mathcal{M}) / \theta_{\delta}$. The presence of a larger number of different congruence classes in the erosion $\varepsilon\left(H_{\mathbb{C}}^{\mathcal{M}}\right)$ reflects in the form of the corresponding quotient lattice $\mathbb{C}(\mathcal{M}) / \theta_{\varepsilon}$, which contains more elements.

This example is interesting because the musical excerpt does not contain only the usual perfect chords, seventh and ninth chords. However, the use of the 7-tet, used here for the simplicity of the illustration, is too limited. It would be even more interesting to use the 12-tet, which would better account for chromatic parts. This is more relevant for atonal pieces of music, where tonality cannot help analyzing and comparing different pieces of music. However, the proposed approach paves the way for such deeper investigations.

\section{Conclusion}

This paper suggests for the first time a possible strategy to approach music information research by combining tools and ideas belonging to two autonomous fields, i.e. mathematical morphology and formal concept analysis. Although some of the concepts described in the paper had already found potential applications in computational music analysis, it is the first attempt at conceiving structural descriptors based on the joint exploitation of these concepts. Introducing congruence relations in lattice-based representations provides a new way of extracting 

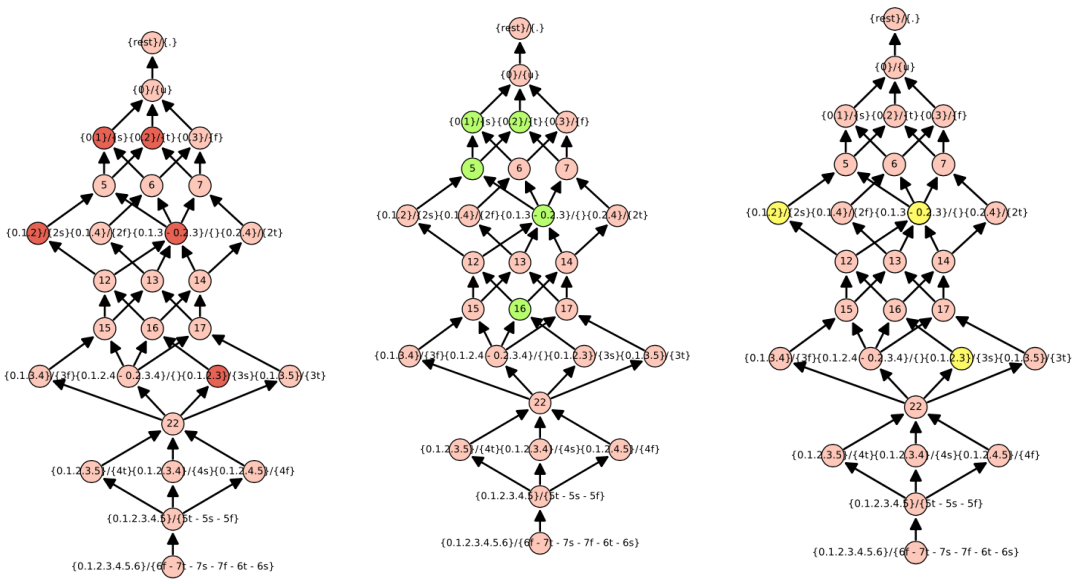

Fig. 5. Formal concepts associated with the harmonic forms found in $H^{\mathcal{M}}$. From left to right: $H_{\mathbb{C}}^{\mathcal{M}}$ (concepts displayed in red), dilation $\delta\left(H_{\mathbb{C}}^{\mathcal{M}}\right)$ (green concepts), and erosion $\varepsilon\left(H_{\mathbb{C}}^{\mathcal{M}}\right)$ (yellow concepts).
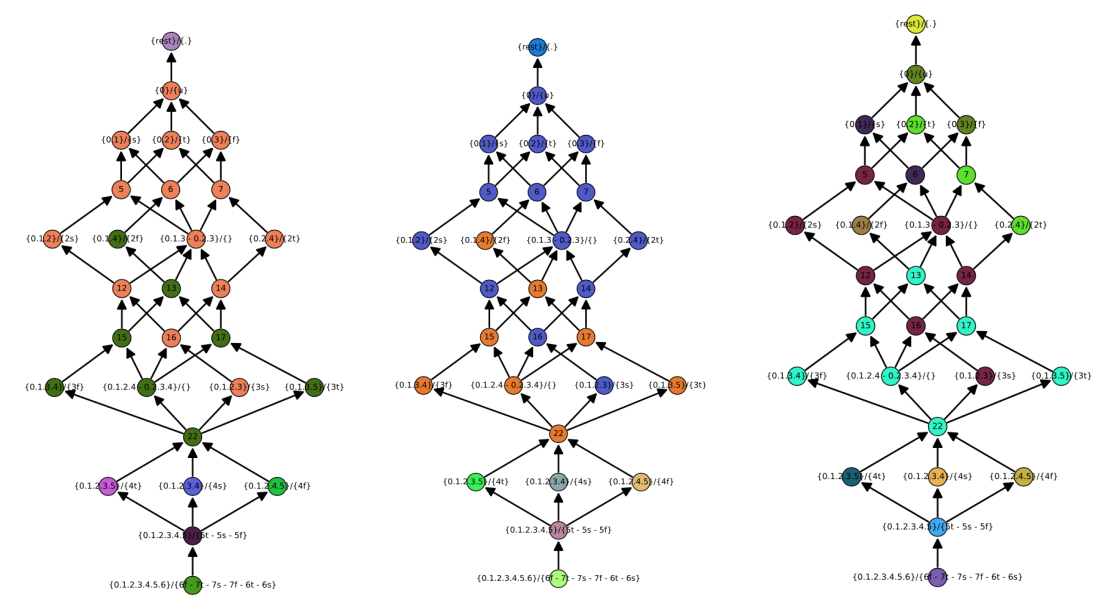

Fig. 6. Congruence relations $\theta, \theta_{\delta}$, and $\theta_{\varepsilon}$ on $\mathbb{C}(\mathcal{M})$ (7-tet) generated by: $H_{\mathbb{C}}^{\mathcal{M}}, \delta\left(H_{\mathbb{C}}^{\mathcal{M}}\right)$, and $\varepsilon\left(H_{\mathbb{C}}^{\mathcal{M}}\right)$, respectively (from left to right).

and summarizing the information contained in a musical piece by preserving the core intervallic structure. The proposed descriptors, particularly suited for atonal and contemporary music which explores the whole space of harmonic forms, are aimed to be used to characterize styles of music, or comparing different pieces of music, using matching between quotient lattices for instance.

Further investigations are needed in order to find meaningful distances (or pseudo-distances) between formal concepts in order to express musically relevant 


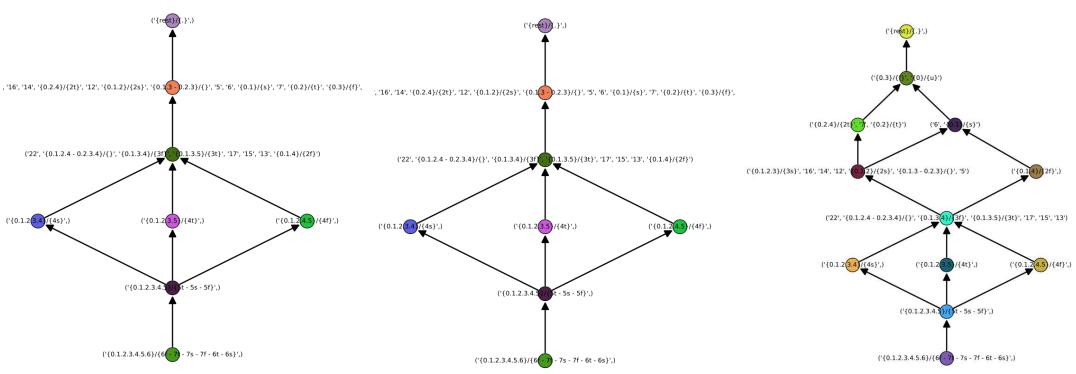

Fig. 7. Quotient lattices. From left to right: $\mathbb{C}(\mathcal{M}) / \theta, \mathbb{C}(\mathcal{M}) / \theta_{\delta}$, and $\mathbb{C}(\mathcal{M}) / \theta_{\varepsilon}$.

morphological operations. At a more abstract level, the question of comparing quotient lattices still remains open in the context of music information research. This goes with the definition of similarities between the descriptors, as for example by establishing whether quotient lattices from two different pieces, or two excerpts of a musical piece, are (or are not) isomorphic. In order to make this comparison computationally reasonable, the compact representation provided by the quotient lattice would be directly exploited. This would clearly provide an assessment of the structural and harmonic similarity between them.

discussion a developper matching de graphes vs de treillis (prenant en compte la structure d'ordre),

\section{References}

1. Atif, J., Bloch, I., Distel, F., Hudelot, C.: Mathematical morphology operators over concept lattices. In: International Conference on Formal Concept Analysis. vol. LNAI 7880, pp. 28-43. Dresden, Germany (May 2013)

2. Atif, J., Bloch, I., Hudelot, C.: Some relationships between fuzzy sets, mathematical morphology, rough sets, F-transforms, and formal concept analysis. International Journal of Uncertainty, Fuzziness and Knowledge-Based Systems 24(S2), 1-32 (2016)

3. Barbut, M., Frey, L., Degenne, A.: Techniques ordinales en analyse des données. Hachette (1972)

4. Birkhoff, G.: Lattice theory (3rd edition), vol. 25. American Mathematical Society (1979)

5. Bloch, I., Heijmans, H., Ronse, C.: Mathematical Morphology. In: Aiello, M., PrattHartman, I., van Benthem, J. (eds.) Handbook of Spatial Logics, chap. 13, pp. 857-947. Springer (2007)

6. Ganter, B., Wille, R.: Formal Concept Analysis. Springer Verlag (1999)

7. Heijmans, H.J.A.M.: Morphological Image Operators. Academic Press, Boston (1994)

8. Heijmans, H.J.A.M., Ronse, C.: The Algebraic Basis of Mathematical Morphology - Part I: Dilations and Erosions. Computer Vision, Graphics and Image Processing 50, 245-295 (1990)

9. Leclerc, B.: Lattice valuations, medians and majorities. Discrete Mathematics 111(1), 345-356 (1993) 
10. Monjardet, B.: Metrics on partially ordered sets-a survey. Discrete mathematics 35(1), 173-184 (1981)

11. Najman, L., Talbot, H.: Mathematical morphology: from theory to applications. ISTE-Wiley (Jun 2010)

12. Neumaier, W., Wille, R.: Extensionale standardsprache der musiktheorie: eine schnittstelle zwischen musik und informatik (2000)

13. Noll, T., Brand, M.: Morphology of chords. Perspectives in Mathematical and Computational Music Theory 1, 366 (2004)

14. Ronse, C.: Adjunctions on the lattices of partitions and of partial partitions. Applicable Algebra in Engineering, Communication and Computing 21(5), 343-396 (2010)

15. Ronse, C., Heijmans, H.J.A.M.: The Algebraic Basis of Mathematical Morphology - Part II: Openings and Closings. Computer Vision, Graphics and Image Processing 54, 74-97 (1991)

16. Schlemmer, T., Andreatta, M.: Using formal concept analysis to represent chroma systems. In: Yust, J., Wild, J., Burgoyne, J.A. (eds.) 4th International Conference on Mathematics and Computation in Music (MCM 2013), Montreal, QC, Canada. vol. LNCS 7937, pp. 189-200 (2013)

17. Schlemmer, T., Schmidt, S.E.: A formal concept analysis of harmonic forms and interval structures. Annals of Mathematics and Artificial Intelligence 59(2), 241256 (2010)

18. Serra, X., Magas, M., Benetos, E., Chudy, M., Dixon, S., Flexer, A., Gómez, E., Gouyon, F., Herrera, P., Jorda, S., et al.: Roadmap for music information research (2013)

19. Serra (Ed.), J.: Image Analysis and Mathematical Morphology, Part II: Theoretical Advances. Academic Press, London (1988)

20. Wille, R.: Restructuring lattice theory: an approach based on hierarchies of concepts. In: Ordered sets, pp. 445-470. Springer (1982)

21. Wille, R.: Sur la fusion des contextes individuels. Mathématiques et Sciences humaines 85, 57-71 (1984)

22. Wille, R.: Musiktheorie und Mathematik. Musik und Mathematik. Springer-Verlag, Berlin-Heidelberg-New York-Tokyo pp. 4-31 (1985)

23. Wille, R.: Restructuring lattice theory: An approach based on hierarchies of concepts. In: International Conference on Formal Concept Analysis. vol. LNCS 5548, pp. 314-339. Springer (2009) 